\title{
Essor du conseil privé et nouveaux profils des ingénieurs agronomes en Argentine
}

Rising of private advisers and new professional profiles of Agronomists in Argentina

\section{Susana Grosso et Christophe Albaladejo}

\section{(2) OpenEdition}

\section{Journals}

Édition électronique

URL : http://journals.openedition.org/economierurale/4083

DOI : 10.4000/economierurale.4083

ISSN : 2105-2581

\section{Éditeur}

Société Française d'Économie Rurale (SFER)

Édition imprimée

Date de publication : 15 octobre 2013

Pagination : 29-39

ISSN : 0013-0559

Référence électronique

Susana Grosso et Christophe Albaladejo, «Essor du conseil privé et nouveaux profils des ingénieurs agronomes en Argentine », Économie rurale [En ligne], 337 | septembre-octobre 2013, mis en ligne le 15 octobre 2015, consulté le 22 avril 2019. URL : http://journals.openedition.org/economierurale/4083 ; DOI : 10.4000/economierurale.4083 


\title{
Essor du conseil privé et nouveaux profils des ingénieurs agronomes en Argentine
}

\author{
Susana GROSSO • UNL Facultad de Ciencias Agrarias, Argentine, Laboratorio AGRITERRIS, \\ susanagrosso@gmail.com \\ Christophe ALBALADEJO • INRA Département SAD, France ; UNLP Facultad de Ciencias Agrarias \\ y Forestales, Argentine, Laboratorio AGRITERRIS, albaladejo@toulouse.inra.fr
}

\begin{abstract}
Cette recherche analyse l'évolution du conseil agricole privé, dans la région pampéenne argentine, à travers une caractérisation des principaux profils de conseillers et des enjeux auxquels ont dû faire face les ingénieurs agronomes qui exercent ce métier. La caractérisation des profils de conseillers d'entreprise et de conseillers technico-commerciaux permet de rendre compte de la consolidation de la modernisation de l'agriculture dans les années 1970-1980. À partir de cette description et à travers les représentations des acteurs concernés, ce travail analyse les changements du secteur et la transformation du métier de conseiller dans les années 1990-2000, au cours desquelles a émergé un nouveau profil : les conseillers en ventes. Les auteurs montrent ici que ce dernier profil n'est pas une spécialisation du profil de conseiller technico-commercial, mais la mise en place d'une logique professionnelle nouvelle, qui remet en question la relation historique construite entre les agriculteurs et les conseillers.
\end{abstract}

MOTS-CLÉS : conseil privé, conseiller agricole, agrofourniture, grandes cultures, Argentine

\section{Rising of private advisers and new professional profiles of Agronomists in Argentina}

This work analyze the evolution of private agricultural advice work in the Argentinean Pampa, through the characterization of the profiles of the advisors and the challenges that agronomist engineers working in this activity have to face. The characterization of the professional profiles of the freelance farms consultants and the technico-commercial consultants is important to understand how significant they were during the periode of consolidation of agricultural modernization in the '70s - '80s. This work studies changes whic occured in agriculture and the modification of the advisor's jobs during 1990 - 2000. Considering the perceptions of the actors themselves, it was been observed that a new professional profile is rising up: the sales advisor. Authors will show that it is not a specialization of the technico-commercial advisor, but a new professional approach that challenges the traditional relationship between farmers and advisors. (JEL: O320)

KEYWORDS: private extension, extension workers, agricultural supply, extensive crops, Argentina

A u cours de ces vingt dernières années, l'Argentine a connu une forte expansion des surfaces en grandes cultures, en particulier du fait de l'adoption d'un paquet technique associant le semis direct aux semences génétiquement modifiées et à l'usage massif de certains produits phytosanitaires et désherbants associés (notamment le glyphosate). Ce système de culture, dans un contexte de libéralisation et de dérégulation de l'économie, a été fortement impulsé par des entreprises de capitaux internationaux responsables de l'agrofourniture et de la commercialisation de la production agricole primaire (Grosso, 2011). Cette évolution a bouleversé le conseil agricole privé.

Le conseil privé s'est développé dans la région pampéenne argentine au cours des années 1950-1960, autrement dit en même temps que le conseil public. Les deux formes de conseil ont été, dès leurs 
origines, directement associées à la modernisation de l'agriculture. Ce nouveau champ d'action s'est construit à partir de trois activités principales :

- le conseil collectif auprès des groupes Consorcios Regionales de Experimentación Agrícola (CREA) ${ }^{1}$ (un mouvement inspiré des groupes du Centre d'études des techniques agricoles français, CETA);

- le conseil technique assuré par des coopératives ou des entreprises locales d'agrofourniture ;

- le conseil individuel aux grandes exploitations en propriété (dénommées estancias). Cette dernière forme de conseil emploie cependant un faible nombre de conseillers.

Les enjeux liés à ces activités ont structuré pendant plus de trente ans les repères du métier de conseiller agricole (un métier presque exclusivement exercé par des ingénieurs agronomes en Argentine et désigné par des termes divers : asesor, extensionista ou tout simplement técnico). Nos travaux de recherche nous ont conduits à distinguer deux profils dominants : le conseiller d'entreprise (asesor de empresas) qui travaille surtout auprès de groupes plus qu'auprès d'individus isolés, et le technico-commercial (técnico-comercial).

Avec les changements survenus ces dernières années dans l'agriculture, un nouveau profil a émergé : le conseiller en ventes (asesor en venta). Dans un premier temps, celui-ci a pu paraître comme une sorte de spécialisation ou de variante du profil technico-commercial. Toutefois, nous montrerons ici qu'il est basé sur une logique professionnelle différente qui remet en question le type de relation établie entre les agriculteurs et les conseillers pendant plus de 50 ans.

Ce travail est le produit d'une réflexion issue de l'analyse d'une trentaine

1. Ils sont rassemblés dans une association nationale : AACREA. d'entretiens approfondis, réalisés entre 2005 et 2008, auprès d'ingénieurs agronomes travaillant dans le conseil privé dans la province pampéenne de Santa Fe en Argentine. Cette province dispose de la plus forte densité d'exploitations familiales capitalisées en zone pampéenne et par voie de conséquence de la plus forte densité de conseillers agricoles et ingénieurs agronomes sur le terrain. Le choix des interlocuteurs (tableau 1) a visé l'ensemble des différents types d'emplois du conseil privé (CREA, coopératives agricoles, entreprises privées de ventes d'intrants, profession liberale) pour deux classes d'âges (25-35 ans et 35-65 ans). Il ne s'agissait pas de constituer un échantillon représentatif, mais de disposer d'un bloc de données couvrant la diversité des emplois afin de générer des hypothèses pertinentes sur les évolutions du métier de conseiller. Ces entretiens semi-directifs, de une à deux heures chacun, se sont centrés sur le récit des pratiques professionnelles des interviewés et ont tous abordé les méthodes mises en œuvre, le contenu du conseil (notamment la place des connaissances scientifiques et des savoirs empiriques), et la relation entre le conseiller et l'agriculteur. Les entretiens ont été traités selon la méthode d'analyse structurale du discours (Demazières et Dubar, 1997). Ces données ont été complétées par l'analyse de documents institutionnels de l'Instituto Nacional de Tecnología Agropecuaria (INTA) ${ }^{2}$, de l'AACREA et du système coopératif afin de mieux comprendre le contexte institutionnel et l'évolution des normes du monde du conseil. Cet ensemble de données nous a permis de générer, dans la province de Santa Fe et plus particulièrement dans l'une des régions de cette province la plus affectée par la transformation

2. Organisme national argentin regroupant les fonctions exercées en France par l'Institut national de la recherche agronomique, les Chambres d'agriculture et les instituts techniques. 
RECHERCHES

Susana GROSSO, Christophe ALBALADEJO

Tableau 1. La constitution de la population enquêtée

\begin{tabular}{c|l|c|c}
$\begin{array}{c}\text { Nombre } \\
\text { d'entretiens }\end{array}$ & \multicolumn{1}{|c|}{ Type d'emplois } & $\begin{array}{c}\text { Nombre } \\
\text { d'entretiens } \\
\text { moins de 35 ans }\end{array}$ & $\begin{array}{c}\text { Nombre d'entretiens } \\
\text { plus de 35 ans }\end{array}$ \\
\hline 6 & Employé d'une cooperative & 1 & 5 \\
\hline 4 & Employé d'une entreprise (vente d'intrants) & 3 & 1 \\
\hline 3 & $\begin{array}{l}\text { Propiétaire/associé d'une entreprise (vente } \\
\text { d'intrants) }\end{array}$ & 0 & 3 \\
\hline 2 & Profession libérale & 0 & 2 \\
\hline 3 & Conseiller CREA & 1 & 2 \\
\hline 2 & Employé de l'INTA (extensionista) & 0 & 2 \\
\hline 10 & Agriculteurs & 1 & 9
\end{tabular}

Source : les auteurs.

des services privés de développement, des résultats que nous considérons comme une série d'hypothèses pour l'ensemble de l'Argentine et qui devront être validées ultérieurement par une enquête d'envergure nationale.

Il faut préciser qu'il existe peu de recherches en Argentine qui analysent le travail des conseillers privés. Presque toutes les recherches portent sur les agents travaillant pour l'État. Certains auteurs apportent des réflexions sur la formation universitaire comme le font Piñeiro (1969) ; Golberg (1988) et Galazzi (2004), et d'autres, analysent les stratégies de conseil (Andrade Franco, 1995), mais en revanche aucun de ces travaux ne s'est concentré sur les pratiques professionnelles de ces agents, hormis nos précédents travaux sur la question (Albaladejo, 2002, 2006). Une récente synthèse bibliographique portant sur plus de 735 travaux de recherche réalisés dans le monde entre 1998 et 2008 et référencés dans les bases $C A B$, Econlit et Web of Science (Faure, Desjeux et Gasselin, 2011), montre clairement que, bien que le thème du retrait de l'État et celui de la « privatisation » du conseil soient parmi les thèmes les plus fréquemment abordés, aucune recherche ne porte sur les conseillers du secteur privé. Pourtant, des auteurs comme Goulet (2011) montrent clairement l'importance en France du conseil pour les firmes de l'agrofourniture qui mettent stratégiquement en avant la fourniture de connaissance en vue d'euphémiser (selon l'auteur) la dimension marchande de leur activité. Ce n'est pas, selon nous, sans conséquence sur le conseil public. Cela explique notamment qu'il soit étudié aujourd'hui avec des méthodes inspirées de courants de recherche ayant été créés au départ pour analyser les activités de service des entreprises (Labarthe et Laurent, 2011). Ce volet de l'activité des firmes a aussi, par contrecoup, profondément bouleversé le métier de conseiller agricole dans son ensemble, tant du secteur privé que du secteur public.

Nous distinguons ici le métier (Piotet, 2002), qui est une activité socialement identifiée correspondant à une identité et un ensemble de savoirs et de pratiques transmis en particulier sur le tas (cas du métier de conseiller agricole), de la profession (Dubar et Tripier, 1998), qui est un système social comportant une reconnaissance officielle (diplôme notamment), des institutions propres (collèges, facultés, etc.) et une mission confiée par la société (accompagner la production agricole). Une profession peut comporter différents métiers et en tout cas les deux notions ne se recouvrent pas forcément. L'emploi est une notion strictement réservée à une fonction rémunérée dans une organisation donnée. 


\section{La naissance du conseil privé et des profils dominants des conseillers}

Presque tous les conseillers agricoles en Argentine sont des ingénieurs agronomes et ce depuis les origines du conseil. Albaladejo a même montré $(2002,2004)$ le caractère central de l'activité de conseil dans la construction de la légitimité professionnelle des ingénieurs agronomes en Argentine. Notre réflexion part ainsi de l'analyse de la pratique professionnelle des ingénieurs agronomes dans leur activité de conseil auprès des agriculteurs, en vue de comprendre l'importance croissante depuis vingt ans du conseil privé en région pampéenne. Pour caractériser cette pratique, nous avons mobilisé trois dimensions de l'activité des conseillers. La première dimension est celle des objets d'étude ou d'action de ces ingénieurs qui sont : la parcelle, l'exploitation et le territoire, reprenant ainsi les catégories d'objet proposées par Sebillotte (2006). La deuxième est celle des intérêts portés à ces objets, autrement dit les manières de procéder et les enjeux qui contribuent à la mise en relation entre l'agronome et ses objets d'étude ou d'action. Nous distinguons notamment trois domaines d'activité qui sont : la recherche, le conseil et l'enseignement, et qui se traduisent par une diversité de pratiques professionnelles et spatiales. Enfin, la troisième dimension est celle de la relation de travail, celle-ci étant entendue comme le type de contrat qui lie le conseiller à celui qui paie le conseil, la durée de ce contrat et la modalité de rétribution économique.

Ces pratiques professionnelles ont été analysées en prenant en compte les transformations au cours du temps : dans les récits de nos interlocuteurs, nous avons tenté de leur demander un récit historique décrivant leurs trajectoires dans le conseil afin de dégager de grandes périodes, et nous nous sommes demandés a posteriori : qu'en était-il autrefois par rapport à aujourd'hui ? Quels défis se présentaient aux agronomes dans les périodes antérieures et à quels challenges doivent-ils faire face aujourd'hui ? Une seconde partie des entretiens portait sur la dynamique de la journée de travail : comment les problèmes à traiter sont-ils détectés ? De quelle façon trouve-t-on des solutions ? Où et avec qui discuter ou échanger pour résoudre les problèmes soulevés par les agriculteurs?

En Argentine, l'ingénieur agronome peut être employé par une organisation économique privée ou être propriétaire (ou associé au propriétaire) d'un cabinet d'études ou d'une «agronomía » (terme qui désigne un commerce de vente d'intrants et de services agricoles), ou encore travailler dans le cadre de l'exercice libéral de la profession.

Pour les ingénieurs agronomes, l'activité de conseil est comprise depuis les années 1970 comme la coconstruction d'un diagnostic, en relation directe avec l'agriculteur, en vue de la recherche d'une solution à une situation problématique. Les trois objets d'étude ci-dessus mentionnés peuvent être tous engagés dans une relation de conseil, sachant que cette activité accorde la priorité à la recherche d'une solution, le plus fréquemment à travers un diagnostic incomplet et en temps limité auquel participe l'agriculteur. Cette interaction vise à construire de nouvelles connaissances ad hoc pour l'action dans une situation concrète jugée problématique.

Dans le cadre privé, le conseil a permis la différenciation de deux profils dominants. Il s'agit tout d'abord du « conseiller d'entreprise » travaillant avec des groupes d'agriculteurs, un profil typique des groupes CREA. Cette expérience a inspiré postérieurement les Grupos de Asesoramiento Integral Cooperativos (GAICO) ${ }^{3}$ et les Acciones Cooperativa de Extensión

3. Promus par la Coopérative Sancor. 
Rural (ACER) ${ }^{4}$, et d'autres, moins répandus dans le territoire. Ce profil a ensuite évolué vers celui de conseiller individuel, mettant en œuvre une vision systémique de l'exploitation. Le second profil est celui du « conseiller technico-commercial », lié aux coopératives et entreprises privées de l'industrie du lait, de stockage de grains et les agronomías. Ce profil relève d'une pratique plus proche du diagnostic-prescription des situations problèmes (selon l'expression de Van den Ban et Hawkins, 1988).

Le profil de conseiller d'entreprise relève surtout du cadre de l'exercice libéral de la profession, tandis que le conseiller technico-commercial s'est développé dans le cadre salarié.

\section{Le conseiller d'entreprise Un profil de référence}

Les conseillers CREA, des pionniers de l'exercice professionnel dans le cadre privé, ont dû faire face au moins à trois grands défis. Premièrement ils ont dû démontrer que la connaissance scientifique à travers le conseil technologique et productif pouvait améliorer la situation des agriculteurs. Deuxièmement ils ont dû motiver ces agriculteurs à travers la méthode groupale, ce qui implique une maîtrise de cette méthode. Troisièmement ils ont dû faire reconnaître leur travail, de telle sorte que l'agriculteur accepte de payer leurs services et les différencie clairement du service public de conseil (extensión) offert gratuitement, notamment par l'INTA.

Le premier de ces défis est à la base des deux autres. Tous nos interlocuteurs analysant, à la lumière de leur expérience, cette époque professionnelle, ont souligné la difficulté de légitimer le conseil dans le milieu agricole : "Je suis sorti de la fac en 1976, mon premier travail a été [...] à

4. Groupes promus par l'Association des coopératives argentines.
San Justo, à cette époque, c'était très dur d'être un ingénieur agronome... parce que la connaissance résidait principalement dans l'expérience empirique, la connaissance qu'appliquait le producteur... et cela produisait des résistances, il était très difficile pour nous de convaincre les producteurs... et nous faisions face à des résistances, de réelles résistances... 》 (Elio, conseiller CREA).

Cette entrée dans le secteur agricole, et donc la reconnaissance, n'était pas facile non plus pour les extensionistas de l'INTA, qui ressentaient tout autant de pressions, bien qu'ils ne dépendissent pas si étroitement «du succès de leur relation de travail avec l'agriculteur pour assurer leur salaire » (Elio). Comme en rend compte l'un d'eux, Juan Carlos : «À ce moment, on portait les efforts sur les aspects sociaux, on travaillait avec les jeunes, les femmes... ces choses, le producteur leur donnait de l'importance et tu pouvais arriver avec un conseil technique si tu étais ami avec lui, mais il n'y avait pas de méthode particulière... maintenant ça a changé. »

Elio, qui a coordonné pendant plus de 20 ans un groupe CREA, commente ce que signifiaient de tels groupes pour un jeune ingénieur récemment diplômé : «J'ai vu dans les CREA non seulement un moyen par lequel nous pouvions accéder ce à quoi nous [les ingénieurs agronomes] aspirions, à savoir nous insérer dans le milieu [des agriculteurs], mais nous pouvions également aller dans le sens du mouvement et être capables de multiplier cette expérience empirique et combiner la connaissance scientifique avec l'expérience des producteurs. »

Cette citation met en évidence que la forme d'insertion des " professionnels $»^{5}$ dans l'activité passait par un travail

5. On appelle «professionnels » en Argentine toute personne ayant une «profession » et donc un diplôme bac+5, dans ce cas il s'agit d'ingénieurs agronomes. 
nécessaire d'hybridation entre les connaissances empiriques de l'agriculteur et les connaissances technico-scientifiques de l'agronome. Donc le conseiller devait observer attentivement le travail de l'agriculteur et voir où et comment la technologie pouvait être un élément d'amélioration du système productif ou du travail agricole. À son tour, cette incorporation technologique devait se traduire dans des résultats économiques facilement mesurables, ou dans une simplification du travail. Cette porte d'entrée, comme cela apparaît dans nos entretiens et dans les documents institutionnels du mouvement CREA que nous avons consultés, a été pendant longtemps « l'incorporation de technologies de coût zéro ». Il s'agissait d'innovations dans la gestion agronomique ou économique. De cette manière, l'amélioration dans les indicateurs physiques (le rendement) a été le premier élément légitimant l'action des agronomes à un moment où l'incertitude économique du pays (forte inflation, dévaluations successives de la monnaie et fortes taxes à l'exportation) rendait pratiquement irrecevable un discours basé sur l'incorporation de technologie à travers l'investissement économique.

Dans de nombreux cas, le paiement des honoraires du conseiller par le groupe d'agriculteurs a été la cause explicite de dissolution des groupes, cette cause pouvant d'ailleurs être réelle ou en cacher d'autres moins avouables, comme le manque de motivation ou des difficultés propres au travail en groupe (Grosso, 1996). Ce qui est certain, c'est que le paiement d'honoraires par les agriculteurs dans notre région d'étude a été (et est encore) un problème récurrent pour les ingénieurs agronomes, et que se légitimer dans le milieu des agriculteurs n'a jamais été une tâche facile. Cette reconnaissance a pu être construite et transmise plus rapidement en créant des Cabinets d'études dont le plus grand développement s'est produit au cours de la décennie 1990 à travers la généralisation de la gestion économico-financière des exploitations.

Le conseiller d'entreprise en Argentine s'est développé sur la base de l'image du modèle professionnel de Parson (1955; cité par Dubar, 2000), entretenant des relations de travail indépendantes, rendant des services à ses clients et, à travers eux, à la société. Les représentations qui dominent ce champ symbolique renvoient à une conception de l'activité professionnelle comme totalement étrangère à d'autres intérêts qui ne soient pas ceux du client. Ils se différencient de l'activité commerciale en mettant en évidence que leurs revenus prennent origine dans la vente des connaissances et non dans la vente d'un produit ou d'un intrant agricole. L'exercice libéral de la profession occupe cependant peu d'emplois actuellement, mais il représente dans l'imaginaire collectif des ingénieurs agronomes un idéal professionnel (Albaladejo, 2011), car il prouve qu'un ingénieur peut vivre exclusivement des connaissances et de l'expertise propres qu'il détient.

\section{Le conseiller technico-commercial Entre technologie et intérêts économiques}

La croissance de ce profil a été favorisée par la mise en place de la Loi 7461/1977, de la province de Santa Fe qui établit que tout achat de pesticides de la part d'un agriculteur requiert une ordonnance (receta, en espagnol) dressée par un professionnel habilité : « le technicien régent », autrement dit un ingénieur agronome formé et inscrit dans une association professionnelle. Nous allons nous concentrer ici sur le monde des coopératives et de stockage de produits des grandes cultures ; nous ne considérerons pas le monde des entreprises laitières qui est un domaine très particulier.

Les conseillers des coopératives ont une relation de travail particulière avec les agriculteurs et leur employeur leur 
demande un grand nombre d'activités diverses. Ces agronomes sont des employés et doivent répondre à la structure administrative de la coopérative, au gérant bien entendu mais aussi - parce qu'il n'est pas un employé ordinaire - au Conseil d'administration. Cette situation de double commande n'est pas simple dans la pratique, et peut conduire à des conflits de travail, personnels, voire d'éthique professionnelle. C'est ainsi, comme le signalent Carricart et Albaladejo (2005), qu'en région pampéenne au cours d'une période de situations financières compliquées et de difficultés de remboursement des dettes des adhérents à leur coopérative (années 1990), les gérants attendaient de l'agronome qu'il les fournissent en informations économiques et financières sur leurs adhérents (en vue du recouvrement), faisant fi de l'obligation de réserve à la base de la relation professionnel-client, qui est à l'origine des professions établies et notamment à celle de leur légitimité (Dubar et Tripier, 1998).

L'un de nos interviewés, Omar, disposant de plus de 20 ans de travail dans une coopérative de la région, se réfère à la relation entre le technicien et le gérant de la manière suivante : "Dans notre coopérative, nous avons toujours eu un appui, on pourrait dire... sans restriction... ce n'est pas qu'ils nous laissent faire ce que nous voulons, mais nous sommes très respectés nous les techniciens... en tant que techniciens, ce qui n'est pas donné parfois. La relation gérant-technicien n'est pas toujours une bonne relation... ».

Une autre particularité est la présence d'un agronome par coopérative, situation qui avec le temps sera modifiée du fait du recrutement d'autres ingénieurs agronomes au regard du développement des activités. Dans ces activités, il faut d'ailleurs distinguer celles de type technique et d'autres plus administratives, bien que parfois dans la réalité une telle séparation ne soit pas aussi simple.
Parmi les tâches techniques se trouvent le conseil technique aux producteurs, l'organisation d'instances collectives d'information et le conseil direct à la coopérative. C'est ainsi que l'agronome a généralement la responsabilité de l'animation d'un groupe d'adhérents qui fonctionne sur le mode des CREA, ou effectue des tâches de coordination du groupe des jeunes (moins de 35 ans) de la coopérative.

Le conseil technique consiste en une série de tâches diverses où ce qui est en jeu est la connaissance agronomique de l'ingénieur. Il comprend des consultations en bureau (généralement le matin) focalisées sur un problème formulé par l'agriculteur au niveau de l'objet parcelle ou de l'exploitation : Quelle variété semer? Quel engrais utiliser ? Comment contrôler des adventices ? Bien entendu, le problème ne peut pas être résolu en bureau, il est par contre enregistré et ultérieurement observé au cours d'une visite sur le terrain. Cette activité présente, du point de vue formel de la discipline, le défaut d'un diagnostic incomplet et d'une approche partielle du problème. Elle conduit à des mécontentements de la part de l'agronome, car celui-ci prétend qu'il n'a accès qu'à une vision partielle et instantanée de la situation agronomique en jeu. Comme cela est généralement désigné dans le jargon professionnel, l'agronome a la sensation d'être « un pompier », autrement dit un sauveur auquel on recourt ponctuellement et subitement quand le problème est déjà là et qu'il est déjà un peu tard pour intervenir.

L'autre type d'activité est l'organisation et la réalisation de journées techniques de diffusion. Avant les années 1990, il était très courant que les conseillers des coopératives organisent une demi-journée en exploitation chez des producteurs démonstrateurs (généralement les membres du Conseil d'administration) afin de montrer aux autres adhérents certains essais ou pratiques culturales innovantes. Actuellement, 
il est plus habituel que ces diffusions soient réalisées par les techniciens des entreprises de l'agrofourniture, qui en profitent pour faire la promotion de leurs produits.

Le troisième type d'activité est le conseil direct à la coopérative. Il est habituellement effectué en fonction de l'approvisionnement des intrants : l'agronome doit recommander quoi acheter, avec quelles caractéristiques ou particularités et en quelles quantités. Ceci n'est pas une activité mineure, car cette prescription détermine le stock de la coopérative, lequel à son tour va conditionner les recommandations aux agriculteurs. Ainsi, quand l'agronome conseille une variété ou un pesticide, cela ne sera pas directement en fonction de l'offre disponible sur le marché, mais en fonction de ce qui est disponible dans la coopérative. Évidemment, les acteurs ont toujours une certaine marge de manœuvre et l'agronome peut recommander un intrant disponible dans un commerce voisin, mais l'adhérant n'a pas forcément la capacité financière d'acheter hors de la coopérative.

Bien qu'elles soient considérées par les conseillers les moins jeunes comme un éloignement de leur cœur de métier, les tâches administratives ont tendance à prendre de plus en plus d'importance, notamment celles d'administration des terres louées et gérées par la coopérative. Parmi ces tâches, la plus importante est celle de régent technique qui établit les ordonnances agronomiques. Une autre, non moins importante - si la coopérative est inscrite en tant que «multiplicateur de semences »-, est le suivi, le contrôle et l'enregistrement de la production de semences. Également, une autre tâche d'administration en émergence qui retombe sur les épaules de l'agronome est la coordination des nouveaux services en équipements offerts aux producteurs. Ce service est apparu du fait de l'augmentation très forte de la consommation en intrants à partir des années 1990 qui a conduit nombre de coopératives à investir dans des équipements coûteux à disposition des producteurs (pulvérisateurs automoteurs notamment).

Ainsi, ce profil technico-économique lié aux grandes organisations économiques des agriculteurs a une place essentielle dans le conseil privé et est depuis les années 1990 en pleine transformation du fait d'une double évolution : celui du métier de conseiller et celui des organisations coopératives.

\section{Les changements dans les années 1990 et leurs conséquences sur le conseil}

Les changements macroéconomiques enregistrés au niveau du pays ont permis aux acteurs les plus puissants du secteur de changer d'échelle de production. Quelques agriculteurs, en particulier les plus modernisés et donc ceux qui maîtrisaient bien les outils de gestion économique et financière (et surtout ceux qui disposaient de capitaux économiques, sociaux et culturels pour faire une telle transformation), ont réalisé un fort processus d'expansion de la production tant avec des capitaux propres qu'avec des capitaux de tiers (notamment à travers de nouveaux instruments financiers comme les fidéicommis, $c f$. Grosso, 2009). En conséquence, on a pu observer de manière rapide un processus de différenciation des agriculteurs ayant des logiques productives différentes, et une concentration de l'utilisation de la terre, mais pas nécessairement de la propriété.

Cela a conduit actuellement à la construction d'une nouvelle structure sociale agricole dans laquelle prédominent des acteurs qui se retrouvent autour de la notion d'agribusiness (Hernández, 2009). Ces transformations sont souvent expliquées par nos interlocuteurs par un changement de pensée lié à un changement de génération dans le secteur agricole. On retrouve cette 
explication dans des documents comme celui de la CEPAL (2005) : «L'agriculteur de la région pampéenne n'est pas le même à partir des années 1990. C'est à présent un chef d'entreprise, d'environ 45 ans, avec une formation académique, avec une autre culture agricole rurale... un producteur qui est professionnalisé et acquiert un haut degré de connaissances d'affaires, qui augmente également l'intervention directe des professionnels et/ou des techniciens dans la direction du processus productif. » Même si cette explication est intéressante, nous nous sommes bien gardés dans nos recherches de considérer cette transformation comme uniquement une question d'arrivée d'une nouvelle génération d'agriculteurs, car nos observations nous montrent bien qu'il y a coprésence de différentes formes d'agricultures et d'agriculteurs sur le terrain. La même prudence est applicable au conseil : les nouvelles formes de conseillers ne remplacent pas les antérieures !

\section{L'agriculteur, devenu entrepreneur, n'est plus fidèle à son conseiller}

Dans cette partie, nous avons procédé au traitement des enquêtes réalisées auprès d'ingénieurs agronomes appartenant au secteur des ventes qui sont entrés en fonction avant les années 1990. Ils ont ainsi eu le temps de se socialiser dans leur métier avant les grandes transformations mentionnées. Le fil conducteur de tous ces entretiens a été la description d'un changement affectant leur activité et qu'ils expliquent par une modification tant du profil des producteurs que de celui des conseillers.

Gustavo, ingénieur agronome propriétaire d'une petite entreprise de vente d'intrants (devenue Centre de services de Monsanto au cours des années 2000) nous parle d'une évolution, dans laquelle on passe en 20 ans de «l'agriculteur» à un «entrepreneur agricole». On est face à «un autre type de situation », dans laquelle les agriculteurs étaient autrefois avant tout des « héritiers » qui « continuaient de faire » en reproduisant voire en étendant l'expérience des parents. Aujourd'hui, il s'est produit pour beaucoup, selon Gustavo, un « retournement technologique» (vuelco tecnológico) qui s'est accompagné d'un changement de mentalités.

Selon lui, un grand nombre de producteurs cherchent actuellement avant tout la rentabilité et, avec elle, le conseil le plus efficace. Ainsi, la dimension personnelle perd son importance dans la relation entre le conseiller et l'agriculteur. L'agriculteur, selon Gustavo et d'autres interlocuteurs, n'est plus «fidèle » : il consulte et compare les avis de différents conseillers avant de prendre une décision, et en particulier pour ce qui est de l'achat des intrants, il n'est plus aussi fidèle qu'il y a 20 ans.

Aujourd'hui, la plus grande taille des exploitations, la recherche du meilleur prix, l'absence de fidélité, l'emploi des silos-sacs ${ }^{6}$ (qui permettent de se passer des services de proximité de stockage), font que les agriculteurs s'éloignent des petits intermédiaires obligeant ceux-ci à faire la différence par rapport aux firmes. Cette différence, ils tentent de la faire grâce à la valeur technique intrinsèque du conseil qu'ils prodiguent.

\section{Le conseiller en ventes De simples vendeurs ou de nouveaux conseillers ?}

L'utilisation croissante des intrants agricoles crée une forte augmentation des

6. Les « silos-sacs » sont des enveloppes de polypropylène de 2 à 3 mètres de diamètre et de 10 à 30 mètres de longueur pouvant contenir jusqu'à 200 tonnes de grains. Selon la condition des grains au moment du stockage, ceux-ci peuvent rester jusqu'à deux ans stockés sur place dans ces silos. On estime actuellement que presque la moitié de la récolte argentine est stockée selon ce système (Bragachini, 2011). 
emplois pour les ingénieurs agronomes liés à la vente des agrochimiques et, avec ces emplois, un nouveau profil semble émerger : celui de conseiller en ventes. Pour avoir une idée précise de l'identité et de l'activité de ce nouveau conseiller, nous avons analysé les entretiens réalisés auprès des plus jeunes d'entre eux, entrés en activité à la fin des années 1990 et début 2000. Nous avons accordé ici une attention particulière à ceux qui sont entrés dans le secteur privé non coopératif.

Deux entretiens nous semblent les plus représentatifs : celui de Darío qui se décrit comme particulièrement engagé dans une identité de vendeur, et celui de Mariano qui, à travers ses représentations, nous a décrit une réalité bien plus complexe et en tout cas relevant plus clairement d'une activité de transformation des compétences.

\section{Un métier de vendeur ou des emplois sous contrainte?}

Darío a passé son diplôme d'ingénieur agronome en 1999, et après six mois d'un premier travail très dur en tant que vendeur dans une entreprise d'approvisionnement en semences, il a trouvé un poste dans une grande et célèbre agronomía de la région qui distribuait divers produits. Darío parle pour son cas de «carrière commerciale». Il affirme que «toutes les connaissances qui lui servent dans son métier actuel ont été acquises sur le tas auprès d'un collègue vendeur plus expérimenté ». Les connaissances commerciales bien sûr, mais aussi toutes les connaissances techniques proviennent de l'expérience et non de l'université. C'est cependant le volet commercial qui définit son activité tout au long de l'entretien : «...J'ai toujours donné la priorité dans mon poste de travail à l'aspect comercial. Il faut se rappeler que celui qui verse notre salaire tous les mois est un commerçant, et nous aussi nous devons être des commerçants, même si les aspects techniques vont avec, comme le tour de parcelles. Le conseil suit, bien sûr, mais je crois que je passe $80 \%$ de mon temps en commercial et $20 \%$ en technique...».

Il dit d'ailleurs que lui et ses pairs ne sont pas des «conseillers [extensionistas] de profession, mais que c'est la partie commerciale qui nous conduit à l'être [...] et la partie du conseil [asesoramiento] est très ponctuelle ». D'ailleurs, il reconnaît que toutes les visites qu'il fait aux producteurs sont motivées par la vente, la livraison d'un produit ou encore pour toucher les chèques et, s'il reste du temps, «nous faisons un peu de technique ». L'objectif de son travail est de «facturer » et, dans son entretien, il compare ses qualités et celles de ses collègues en termes de «potentiel de facturation » (potencial de facturación) : «Je suis principalement évalué par mon niveau de vente». La fameuse double évaluation (par la structure et par les agriculteurs du terrain) qui est si classiquement évoquée par les conseillers publics en Argentine dans la description de leur métier, est ici présentée de façon exacerbée et centrée sur la vente : " C'est un travail usant, et pourquoi il est usant? Parce que tu es en contact en permanence avec des gens, les supérieurs ou le patron et tes clients. Les clients te pressent, ils se font tirer l'oreille pour payer et tu leur dois toujours un sourire [...] et de la part du patron, il y a les rapports à écrire qui doivent lui dire qui tu as vu, ça m'ennuie et je résiste à le faire ».

Cela le conduit d'ailleurs à concentrer son activité sur les plus gros clients et à délaisser les plus petits agriculteurs, ce qui veut dire dans son cas sur les 20 à 25 clients auprès desquels il réalise plus de $80 \%$ de ses ventes, parmi lesquels 10 à 15 ne sont pas des agriculteurs mais sont eux-mêmes des commerçants d'intrants. $\mathrm{Si}$, comme certains auteurs l'ont montré (Laurent et al., 2002), on prend en compte que l'identité professionnelle est en grande partie déterminée par le public cible des 
conseillers, cette considération marque un changement de taille dans le métier.

Mais il semble que les conseillers comme Darío décrivent plus une transformation de l'emploi que du métier ${ }^{7}$. Emploi qui d'ailleurs est dans l'ensemble une « souffrance » au sens où le décrit Desjours (1998) pour d'autres contextes. En effet, ils expriment généralement le souhait frustré de travailler différemment. De fait, Darío distingue avec soin deux agriculteurs, qu'il décrit comme des amis, qui sont les seuls chez qui il prodigue des conseils techniques. Bien qu'ils ne soient que deux, et bien que ses visites concernent généralement la vente, ils jouent un rôle important dans son discours, dans son identité professionnelle et dans sa façon de s'évaluer, car c'est dans ces deux exploitations qu'une relation de confiance lui permet de tester sa capacité à formuler des avis techniques plus globaux. Darío nous dit : «Je ne manque pas d'envie d'avoir la possibilité d'avoir plus de clients [sous-entendu d'autres personnes que ses deux «amis »], c'est-à-dire faire plus de conseil. Mais étant donné les circonstances, le moment que nous traversons, la conjoncture, si l'on n'est pas un vieil ingénieur agronome qui a déjà son carnet de clients, c'est quasiment impossible pour un jeune de faire $d u$ conseil [...] c'est pour ça que je dois me contenter de ce que je fais. » Il est d'ailleurs sans ambiguïté sur ses motivations actuelles : «Je fais ça pour de l'argent », nous a-t-il dit presque pour se justifier.

7. Selon les termes définis par Paugam (2000), l'emploi renvoie à une définition des fonctions dans l'organisation et pour l'organisation dans laquelle la personne travaille (définition procédurale des qualifications) et au statut du contrat de travail. Le métier renvoie à une utilité sociale du travail dépassant l'organisation qui emploie cette personne et à des valeurs et normes définies par une communauté de pairs. Le métier va de pair avec une définition patrimoniale des qualifications : l'individu en est détenteur et peut les transférer d'une organisation à l'autre s'il change d'emploi.
D'ailleurs cette activité ne lui laisse pas le temps d'aller à des formations continues dans son domaine : "Remplir ses obligations par rapport au patron et aux clients prend tout le temps et ne nous laisse pas le temps de faire des choses importantes comme la formation, on ne lui [à la formation] accorde pas le temps qu'on devrait. »

\section{Description d'un nouveau profil de conseiller...}

D'autres discours ont pu être relevés chez ces plus jeunes ingénieurs de la vente, moins marqués par une souffrance de l'emploi et réussissant à décrire une façon originale de pratiquer le métier de conseiller.

C'est le cas en particulier de Mariano qui a fini ses études d'ingénieur en 2000 et qui, après avoir travaillé dans un commerce de vente de légumes et avoir à plusieurs reprises été recruté en main-d'œuvre occasionnelle en tant qu'enquêteur-recenseur des agriculteurs et surfaces en grandes cultures d'une petite région pour le compte de grandes compagnies (Monsanto, Pioneer), a finalement trouvé un emploi chez l'un des distributeurs officiels de Pioneer. Bien que Mariano se décrive comme « un bon vendeur » et en donne avec fierté les raisons à de nombreuses reprises, tout son discours vise à décrire une activité professionnelle, et non une activité de commerce, mais d'un type un peu spécial.

Tout d'abord il ne décrit pas son employeur comme un simple commerçant : «Nous pourrions vendre un peu de tout... mais ce n'est pas ce que nous faisons... parce que quand on a moins de choses dans la tête on est plus efficace. Par exemple, je vois les gars des autres entreprises qui vendent des inoculants, fertilisants, humidifiants, fils de fer, piquets, semences, produits agrochimiques... [...] Nous, on n'a pas tout ça en tête, quand on va le voir [1'agriculteur] pour le maïs, pour 
la luzerne ou pour le sorgho, on sait à quel moment de l'année y aller et on sait parfaitement pourquoi on y va...». Le statut d'exclusivité de Pioneer donne d'ailleurs dans ses propos une certaine supériorité à l'employeur qui le situe au-dessus de la mêlée de la concurrence commerciale : "Quand je vais voir un agriculteur, j'y vais avec le maillot de Pioneer, c'est-àdire en exclusivité, et ça lui met plus la pression, pour le dire comme ça, tu arrives plus facilement à pénétrer que n'importe quel autre qui vend plein d'autres choses...».

Ce concept de «pénétration » est central dans le discours de Mariano et de nombre de ses collègues. Il est à la fois un mélange d'évaluation commerciale (pénétration dans son sens le plus strict signifie chez Mariano le pourcentage d'agriculteurs qui achètent Pioneer parmi ceux à qui il rend visite...), et d'évaluation de la compétence technique du conseiller. En effet, selon Mariano, Pioneer estime que la pénétration est en relation directe avec la « différenciation »: se différencier de la concurrence par la qualité de l'appui technique. À la question d'ailleurs de savoir comment il est évalué, Mariano ne parle pas de « facturation », mais plutôt de « différenciation », autrement dit par son travail de conseil technique : «Pioneer cherche la façon de se différencier, sans doute parce qu'il y a tellement de concurrence que c'est la seule façon de marquer la différence. Et cela s'obtient à travers les instruments que fournit Pioneer. » On retrouve ici les stratégies des firmes de semoirs en semis direct observées par Goulet (2011) en France.

Mariano passe une partie importante de son discours à décrire ces instruments de travail qu'il mobilise, en particulier les « essais » (los ensayos), les « suivis de parcelles » (seguimientos) et les « rapports sur les cultures » aux producteurs (informes). Ces instruments sont clairement ceux de l'agronome, et ils sont les axes de construction d'une relation de confiance avec l'agriculteur qui passe donc par le développement d'une activité de conseil technique et est censée avoir une incidence sur la «pénétration » commerciale. De plus, Mariano souligne l'importance qu'accorde Pioneer à la formation continue de ses conseillers.

Ces instruments semblent les indices d'un nouveau type d'activité chez les conseillers, qui ont plus affaire à des agriculteurs absents de la partie agronomique de terrain et qui substituent en quelque sorte leur présence dans leurs propres champs par celle du conseiller et par les observations et le diagnostic de celui-ci.

Mariano se définit lui-même non pas comme un vendeur mais comme un conseiller (asesor) avec deux volets dans son activité : le conseil commercial et le conseil agronomique. On comprend alors que son activité de conseil agronomique (il dit aussi parfois conseil technique) joue un rôle essentiel dans sa façon d'exercer la profession (d'ingénieur agronome), même si Mariano nous dit, comme Darío, qu'elle ne lui prend que $20 \%$ de son temps tandis que l'activité commerciale en prend $80 \%$.

Autre élément essentiel de la façon d'exercer la profession de Mariano : son public cible. Il ne va pas voir que les grands, autrement dit que ceux qui sont les plus intéressants commercialement. « J'ai beaucoup d'exploitants cultivant moins de 100 ha de maïs. [...] Avec les petits c'est un autre type de conseil, on ne peut pas faire des essais, mais tu te retrouves avec d'autres choses...». Cette assise sociale donne de la noblesse à son activité de conseil et donc une légitimité

8. Pionner caractérise les agriculteurs selon l'importance de la surface de maïs qu'ils cultivent sans délaisser totalement ceux qui cultivent moins de 100 ha de cette culture, selon ce que nous explique Mariano. 
professionnelle qui semble une composante essentielle au reste de son activité.

Malgré tout, Mariano note que la profession d'ingénieur agronome, "même si elle existe encore », nous dit-il (cette précision spontanée semblant indiquer qu'il y a un doute exprimé dans sa communauté de pairs), passe toujours après les aspects commerciaux : "Aujourd'hui, si je dois aller voir deux agriculteurs mais n'ai le temps de n'en voir qu'un, pour si malade que soit le maïs de celui chez qui je dois faire le suivi technique et à qui j'ai déjà vendu, je privilégierais quand même celui à qui je peux vendre..., car sans doute qu'on gagne par cet aspect-là [le conseil] mais le bénéfice vient des ventes. » $\mathrm{Il}$ le regrette notamment : "Sur un plan éthique, j'aimerais aller voir la parcelle de celui qui m'a acheté le maïs et voir s'il est malade... la partie agronomique... mais je dois m'incliner vers la partie commerciale [...] je mets en place mon agenda à partir de la partie commerciale, mais dans l'année il y a quand même des moments qui sont nettement agronomiques, qui sont des moments fantastiques, par exemple de septembre à maintenant [décembre] c'est tout agronomique. Mais la partie terriblement commerciale commence en avril-mai... ». D'ailleurs, chacun de ces instruments peut être compromis par une attitude trop commerciale. C'est déjà le cas des essais, la concurrence s'étant emparée de l'idée et on trouve déjà, selon Mariano, des agriculteurs chez qui sont menés trois à quatre essais différents, avec le risque de les lasser.

La « différence », Mariano ne la fait pas seulement avec les outils de l'agronome développés par l'entreprise, mais aussi par la relation personnelle, attitude traditionnelle des conseillers en Argentine (extensionistas) : "La décision d'un producteur de m'acheter Pioneer passe à 80 ou $85 \%$ par la façon dont je suis avec lui, par l'amitié, par la relation, plus que par les qualités du produit... ».
Ainsi, l'évolution des profils des conseillers vers plus d'activité commerciale est quasi générale et a des conséquences très fortes sur les méthodes de travail et les identités professionnelles. Cette évolution n'est pas du tout vécue de la même manière selon les classes d'âge des conseillers. Certains le vivent mal et de façon très dichotomique, tandis que d'autres tentent d'incorporer la dimension commerciale à leur manière de construire leur identité et leur légitimité professionnelle.

$$
\begin{gathered}
* \\
* *
\end{gathered}
$$

L'analyse des discours des conseillers agricoles travaillant en zone pampéenne de grandes cultures sur la transformation de leur activité montre que le métier du conseil a joué un rôle central dans la légitimation de la profession d'ingénieur agronome en Argentine et que ce métier a très tôt défini deux profils dans le privé (conseiller d'entreprise et conseiller technico-commercial) et un profil unique dans le public (extensionista de l'INTA).

Mais, depuis les années 1990, nous assistons à de fortes transformations dans le champ du conseil privé, en particulier la vente est devenue la partie essentielle de l'activité de la plupart des conseillers de ce secteur. Pourtant, même si fortement minoritaire dans le temps d'activité, l'activité de conseil reste au cœur de la construction de la légitimité professionnelle de l'ingénieur agronome et un élément important de la stratégie des firmes dans leurs relations aux agriculteurs.

Nous avons pu étudier également l'émergence d'un nouveau profil : le conseiller en ventes. Ce profil, bien qu'il puisse être interprété en première analyse comme une simple spécialisation de l'un des deux profils antérieurs (celui de technico-commercial), il se présente comme la possible représentation d'une autre manière de faire du conseil, au moins dans le contexte d'étude. 
Il considère le producteur comme un client, et pose la réussite commerciale comme l'indicateur de l'efficience du travail, pas seulement parce que le salaire de ce conseiller comporte toujours un pourcentage sur les ventes des intrants. D'autres recherches sont nécessaires pour identifier si nous sommes simplement face à l'émergence d'un nouveau secteur d'emplois pour les ingénieurs agronomes ou face à un nouveau profil dans le métier du conseil, avec ses outils, ses représentations, son vocabulaire propre, son nouveau public cible (des agriculteurs se pensant en entrepreneurs d'affaires agricoles et non plus en entrepreneurs agricoles et encore moins en agriculteur).

La réponse à la question des nouvelles formes de légitimation du conseil, ses nouveaux outils, ses nouveaux profils de métier, requiert un élargissement du cadre d'analyse pour évaluer la possible émergence de ce qui se réclame être un «nouveau paradigme du développement » dans les grandes cultures avec ses institutions, son discours et ses représentations, ses propres connaissances produites par un secteur de la recherche, etc.

À cette étape, nous pouvons détecter que ces changements conduisent à des tensions croissantes entre ce qui définit le cœur de la profession de conseiller, construit sur l'idéel des «professions classiques » (médecins et avocats notamment : en particulier une relation experte et de confiance auprès d'un client), et la réalité actuelle du travail dans laquelle la plupart des emplois sont fournis par des entreprises de l'agrofourniture. Cela se traduit par des contradictions touchant à la connaissance et à l'identité socioprofessionnelle. Dans ce contexte, l'accès aux emplois, peu nombreux, qui restent en relation au conseil privé non commercial est devenu un objectif pour nombre d'ingénieurs agronomes et le rêve de la plupart des étudiants d'agronomie.

Ce travail a bénéficiéd'une aide del'Agence nationale de la recherche et de l'AIRD dans le cadre du programme SYSTERRA, portant la référence ANR09-STRA-04 et du département SAD de I'INRA dans le cadre de la convention AGRITERRIS.

\section{RÉFÉRENCES BIBLIOGRAPHIQUES}

AACREA (1973). ¿Qué es un CREA? Buenos Aires, AACREA, $12 \mathrm{p}$.

Albaladejo C. (2002). Les fonctionnaires et le développement rural en Argentine depuis 1991 : entre la profession et le territoire, entre l'État et la ville. Paris, Autrepart, Revue de Sciences Sociales au Sud, ${ }^{\circ} 23$, septembre, p. 43-56.

Albaladejo C. (2006). Le déclin institutionnel du développement agricole en Argentine : paroles d'agents en quête d'identités. In Baré J.-F. (éd.), Paroles d'experts. Comment les intervenants du développement pensent leur action, Paris, Karthala, p. 162-199.

Albaladejo C. (2011). Transformations du conseil agricole en région pampéenne argentine et recomposition de la profession d'ingénieur agronome. Paris, Cahiers Agricultures, vol. 20, n ${ }^{\circ}$ 5, p. 387-94.

Andrade Franco P. (1995). Asesoramiento grupal y su relación con la integración, capacidad empresarial y productividad en grupos de productores lecheros. Entre Ríos, Argentina. Thèse de Magister Scientiae, Posgrado en Extensión Agropecuaria. Rafaela, FAVEINTA, $165 \mathrm{p}$.

Bragachini M. (2011). Desarrollo industrial de la maquinaria agrícola y agropartes en Argentina. Santa Fe (Argentina), Documento de trabajo EEA INTA Manfredi, $34 \mathrm{p}$.

Carricart P., Albaladejo C. (2005). Reflexiones críticas sobre los espacios emergentes: las cooperativas agropecuarias y los espacios rurales en la región pampeana argentina. 
In Benencia R., Flood C. (eds.), Trayectorias y contextos. Organizaciones rurales en la Argentina de los noventa, Buenos Aires (Argentina), La Colmena, p. 49-88.

CEPAL (2005). Análisis sistémico de la agriculturización en la Pampa húmeda argentina y sus consecuencias regionales extrapampeanas: sostenibilidad, brechas de conocimiento e integración de políticas. Santiago de Chile, CEPAL, Serie 118, 65 p.

Demazière D., Dubar C. (1997). Analyser les entretiens biographiques. L'exemple des récits d'insertion. Paris, Nathan, 349 p.

Desjours C. (1998). Souffrance en France. La banalisation de l'injustice sociale. Paris, Seuil, coll. « Points », 223 p.

Dubar C. (2000). La socialisation. Construction des identités sociales et professionnelles. Paris, Armand Colin, coll. «U », 276 p.

Dubar C., Tripier P. (1998). Sociologie des professions. Paris, Armand Colin, coll. « U Sociologie », $256 \mathrm{p}$.

Faure G., Desjeux Y., Gasselin P. (2011). Revue bibliographique sur les recherches menées dans le monde sur le conseil en agriculture. Paris, Cahiers Agricultures, vol. 20, $\mathrm{n}^{\circ} 5$, p. 327-342.

Galazzi E. (2004). Formación del ingeniero agrónomo en extensión rural. Bases de una propuesta para la Universidad Nacional del Sur. Memoria de Maestría en Extensión Agropecuaria, Esperanza (Argentine), Universidad Nacional del Litoral, 193 p.

Golberg A. (1988). La dimensión ambiental de la Agronomía. Santa Rosa (Argentina), Documento de trabajo, Facultad de Agronomía, Universidad Nacional de La Pampa, 12 p.

Goulet F. (2011). Accompagner et vendre. Les firmes de l'agrofourniture dans l'innovation et le conseil en agriculture. Cahiers Agricultures, vol. 20, n 5 , p. 382-386.

Grosso S. (1996). La perdurabilidad de los grupos de asesoramiento agropecuario. Esperanza (Argentine), Memoria de cientibeca,
FAVE, Universidad Nacional del Litoral, $97 \mathrm{p}$.

Grosso S. (2009). Les pools de culture : diversité des combinaisons financières et productives. DÉMÉTER 2010, Dossier Argentine, Paris, Club Déméter, p. 223-254.

Grosso S. (2011). Les agronomes de terrain face a une nouvelle agriculture pampéenne : stratégies pour la reterritorialisation des connaissances agronomiques. Thèse de Doctorat de l'Université de Toulouse II, 401 p.

Hernández V. (2009). La ruralidad globalizada y el paradigma de los agronegocios en las pampas gringas. In Gras C., Hernández V. (eds.), La Argentina rural. De la agricultura familiar $a$ los agronegocios, Buenos Aires, Biblos, 345 p.

Labarthe P., Laurent C. (2011). Économie des services et politiques publiques de conseil agricole. Cahiers Agricultures, vol. 20, $\mathrm{n}^{\circ} 5$, p. 343-351.

Laurent C., Cerf M., Pasquier C. (2002). Le conseil en agriculture : un investissement immatériel entre développement sectoriel et développement territorial. Paris, Géographie, Économie, Société, n 4, p. 131-153.

Paugam S. (2000). Le salarié de la précarité. Les nouvelles formes de l'intégration professionnelle. Paris, Presses universitaires de France, coll. «Le lien social », 437 p.

Piñeiro A. (1969). Reflexiones sobre el ejercicio y la enseñanza de la Agronomía. Esperanza (Argentine), Conférence à la FAVE, Universidad Nacional del Litoral, 26 septembre $1969,19 \mathrm{p}$.

Piotet F. (2002). La révolution des métiers. Paris, Presses universitaires de France, coll. « Le lien social », 362 p.

Sebillotte M. (2006). Penser et agir en agronome. In Doré T., Le Bail M., Martin P., Ney B., Roger-Estrade J. (eds.), L'agronomie aujourd'hui, Versailles, Éditions Quae, p. 1-21.

Van den Ban A., Hawkins H. (1988). Extensión Agraria. Zaragoza (Espagne), Editorial Acribia, 340 p. 